\title{
Testing the modified dependence of the radiative strength function on different excitation energies in the light nucleus ${ }^{28} \mathrm{Al}$
}

\author{
${ }^{1}$ D. Knezevic, N. Jovancevic ${ }^{1}$, A. M. Sukhovoj ${ }^{2}$, L.V. Mitsyna ${ }^{2}$ \\ ${ }^{1}$ University of Novi Sad, Faculity of Science, Department of Physics, Novi Sad, Serbia \\ ${ }^{2}$ Joint Institute for Nuclear Research, Dubna, 141980, Russia \\ E-mail: nikola.jovancevic@df.uns.ac.rs
}

Received: December 29, 2017

\begin{abstract}
The spectrum of random functions of level density as well as radiative strength functions of dipole E1- and M1-transitions of ${ }^{28} \mathrm{Al}$ were determined. Obtained functions can reproduce very precisely the intensity of the two-step cascade following the radiative capture of thermal neutrons for a given energy of the primary transitions. The density of the observed intermediate levels can be reproduced correctly using the mean value of these functions. In this work we proposed a new hypothesis about the dependence of radiative strength functions for gamma-transitions in heated nucleus on the energy of excited levels. The results provide a solid basis that this new hypothesis allows to get realistic estimation on the parameters of nuclear structure in any nucleus, including the light ones.
\end{abstract}

Key words: Neutron resonance, two step gamma cascades, level density, radiatve strength function.

\section{Introduction}

The determination of accurate values for the excited nuclei level density and radiative strength functions is one of the most important tasks in low energy nuclear physics. Trustable experimental values of these parameters are necessary for the study of the fundamental properties of the nuclear structure. For example, the step-like structure in the level density provides information about the phase transitions in heated nuclei and the influence of different type of resonance wave functions on radiative strength function for the $\gamma$ decay process. Moreover, accurate experimental values of the level density and the radiative strength function are very important for applications as the analysis of astrophysical reactions, the production of medical isotopes, reactor technology, the production of rare isotope beams, etc. 
The development of theoretical models needs a set of experimental information for the excited levels density (with given quantum numbers) and for the values of the partial radiative widths of all possible decay channels. If those data are available, the theoretical calculations can give a correct interpretation of the dynamics of the nuclear transitions, in a broad variety from the simple low-lying levels (e.g., quasiparticle or phonon structure) to the very complex compound-states.

The quality of the model-based description of all the parameters for the neutron resonance gamma-decay, for example, depends entirely on the precision of the experimental data. Hence, it is very important to minimize the overall experimental uncertainty and, accordingly, to minimize the possible misrepresentation in analysis of the observed process, mostly as a result of the use of certain assumptions and the related hypothesis. The ability to get an accurate solution substantially increases when in the measurement are employed nuclear spectrometers, having $F W H M \ll D_{\lambda}$ for all energy spaces $D$ between the initial nuclear levels $\lambda$.

In this situation a fundamental experimental problem is to search for the connection between the emission probability $\Gamma$ of the reaction product and the excited levels densities $\rho$. The sum of the branching ratios $B_{r}\left(B_{r}=\Gamma_{\lambda i} / \Gamma_{\lambda}\right)$ for partial $\Gamma_{\lambda i}$ and total widths $\Gamma_{\lambda}$ (if there are no competing processes) is equal to one and does not depend on the absolute values of the levels density $\rho$ and the partial widths $\Gamma$. However, branching ratios of any level are determined by the sum of the partial widths and consequently they are dependent on the level density. So, to give an accurate description of the dependence between the measured values (intensity of the emitted spectrum of particles observed in the reaction), the excited levels density and the partial radiative widths is one of the most important tasks in the process of estimation of those parameters values.

In this work we proposed a new and a modified form of the dependence for the radiative strength function of the excited level density, based on the analysis of the experimental data and the existing models of the level density as well as the partial gamma-widths. This new approach was tested experimentally by the estimation of the most probable mean values of the level density and the radiative strength function of ${ }^{28} \mathrm{Al}$ compound-state gamma-decay. To accomplish this task, the Dubna two-step gamma cascade method was used [1, 2].

\section{Theoretical considerations \\ 2.1. Current state of the experiments designed for the determination of $\Gamma$ and $\rho$}

The information about the properties of the excited nuclei can be extracted only by the measurement of the spectra (cross-sections) $S$ of the observed reaction product, and the subsequent analysis based on some existing functional dependencies between $S$ and the parameters $\Gamma$ and $\rho(S=\Psi(\Gamma, \rho))$. Such experiment can be performed measuring the reaction products spectra with a single detector ("one-step" reaction [3]), or by coincidences between two detectors ("two-step" reaction $[4,5]$ ).

The first of these two experimental techniques was used up to now for the analysis of the spectra and the cross-sections of evaporative nucleons $[3,6]$ and full gamma spectra $[7,8]$. The second one was applied in the spectroscopy measurements of two photons $[3,4]$ successively emitted after the neutron capture. Comparison of the $\Gamma$ and $\rho$ values obtained in the analysis of the experimental data collected in one- and two-step reactions, makes 
possible to identify the main sources of the systematic experimental uncertainties, to estimate the uncertainty values and to compare them if different methods were applied in the study of the same nucleus.

\subsubsection{Spectra of evaporative nucleons}

The level density can be obtained from the evaporative nucleons spectra by the use of this method only if the value of $\Gamma$ is known. The numerical value of the parameter $\Gamma$ was calculated until now from the relativly rudimentary optical model. The agreement between the calculated cross-section and the experimentally determined one can be used for the validation of the obtained results. However, this method does not take into consideration that the experimentally measured cross-section (spectrum) is determined only by the absolute value of the product $\Gamma \cdot \rho$, and not by the absolute values of the individual terms $\Gamma$ and $\rho$. One of the consequences (as can be seen comparing the data measured by this method $[3,8]$ with the two-step reaction analysis $[4,5]$ ), is that the obtained values for the level density in the energy range around the threshold of the second gap for nucleons Cooper pairs are overestimated at least by 5-10 times [9].

\subsubsection{Spectra of the primary quanta of cascades depopulating different energy exited states}

The total intensity of the spectra of the first generation of cascade quanta [7] does not depend on the absolute values of $\Gamma$ and $\rho$. Moreover, the absolute value of the function describing the dependence of the mentioned parameters $\Gamma$ and $\rho$ on the energy of the gamma-quanta and the energy of the excited level has no significant influence on the total gamma spectra (the sum of the gamma energies of cascade photons depopulating some level is absolutely independent from $\Gamma$ and $\rho \ddot{I}$ ) $)$. The mean quadratic variation of the different forms of the full gamma-spectra calculated employing various realistic representations of $\Gamma=f\left(E_{\gamma}\right)$ and $\rho=\varphi\left(E_{e x}\right)$ does not exceed $30 \%$, in the best case, [10] (where $E_{e x}$ is the exited level energy).

Fig. 1 shows the gamma rays spectra [11] following the inelastic scattering of ${ }^{3} \mathrm{He}$ on the ${ }^{45} \mathrm{Sc}$ isotope. The intensive and well-resolved low energy peaks registered in the "first generation" and the in "higher-generation" spectrum are produced, in most cases, only by second or higher cascade quanta [11]. These gamma peaks appear in the "first generation" spectra only due to an error of the specific techniques used. Specifically, this is a consequence of the non-compliance of the basic condition of the used technique [7]. The spectrum of the gamma radiation emitted after the decay of the levels for a given excitation energy is initialized by the beam of the charged particles and described by the one particular set of quantum numbers. This spectrum should be exactly the same as the spectrum measured in the experiment where observed levels are populated by the transitions from the high-lying energy levels. As a result, the systematic error of the "first generation" spectra can exceed $100 \%$ in low energy photon region. The increase of $E_{\gamma}$ can reduce this error by some unknown degree. This fact is not mentioned in the evaluation [12] of the error appearing in this approach [8]. 


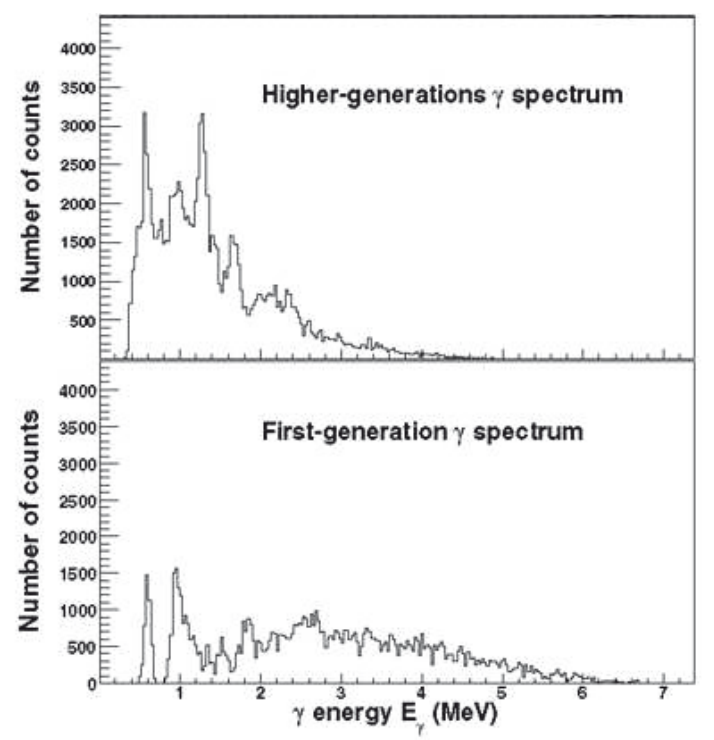

Figure 1: Gamma-ray spectra in the ${ }^{45} \mathrm{Sc}\left({ }^{3} \mathrm{He},{ }^{3} \mathrm{He} \gamma\right){ }^{45} \mathrm{Sc}$ reaction [11] for the first and next cascade quantum.

\subsubsection{Two-step cascade quanta}

The absolute intensity of the cascades $I_{\gamma \gamma}=\Psi(\Gamma, \rho)$, which can be measured by ordinary HPGe-detectors for a limited number of final levels, is defined by the inverse absolute value of the level density and by the form of the strength function $K=f\left(E_{\gamma}\right)$ [13]. Thereby, the relationship between the experimental values of $I_{\gamma \gamma}$ and the unknown functions $\Gamma$ and $\rho$ is always nonlinear, for all intervals of the excitation energy. The current experimental methods allow to determine only an interval for the possible values of $\Gamma$ and $\rho$ reproducing the measured intensity of the cascades $I_{\gamma \gamma}$. Even in the limit of zero statistical errors of the experimental values, $\Gamma$ and $\rho$ could not be unequivocally determined [14].

All the methods listed above have also common sources of systematic errors.

1. There is no practical model of the decay of the nuclear excited levels, for both nucleon and radiation channel, suitable for the analysis of the experiment. Such a model should be able to take into account explicitly the coexistence and the interactions of the boson and the fermion components of the nuclear matter.

2. It is necessary that the model considers the dependence of the partial widths $\Gamma$ on the wave function for both the initial and the final level when the reaction product of a specified energy is emitted.

All the three techniques listed above, without exception, requires an additional, methodically independent, experiment. That experiment should produce a non-degenerate system of equations which can describe the relation between the measured spectrum and the values of the parameters $\Gamma$ and $\rho$.

The influences of the mentioned sources of systematic errors, which are most significant for one-step reactions, can be essentially reduced in the two-step reaction experiment. Therefore, it is necessary to develop a modern model able to describe and predict possible changes of the nuclear properties caused by different excitation energies. This model would 
require data from two-step reaction experiments.

\subsection{Status of the current models of the level density and the partial gamma-widths}

In modern theoretical views, for example for the quasiparticle-phonon model of the nucleus, the partial emission width is determined by the coefficients of the wave function components for both decayed and excited level [15]. The actual values of the partial widths are specified by the degree of the fragmentation for the different nuclear states with a fixed number of quasiparticles and phonons. The level density directly determines it, since the $\rho$ value is defined by the degree of the fragmentation of all the possible states of the nucleus having energy lower than the excitation one.

Currently, the radiation strength function of the dipole gamma transitions for a nucleus with mass $A$ can be expressed as:

$$
k_{\text {standard }}=\Gamma_{\lambda i} /\left(E_{\gamma}^{3} A^{3 / 2} D_{\lambda}\right)
$$

where $E_{\gamma}$ is the energy of the emitted gamma quanta, $A$ is the atomic mass, $D_{\lambda}$ is the density of the decaying level and $\Gamma_{\lambda i}$ represents the partial width of the nucleus transition from the $\lambda$ to the $i$-th level.

The expression above takes into account the dependence of the partial radiative widths only from the density $\rho_{\lambda}=D_{\lambda}^{-1}$ of the decayed high-energy levels, such as the neutron resonances. However, the possibility that the partial radiative widths can be a function of the density of the intermediate levels of the heated nucleus having sufficiently high energy is not taken into consideration by Eq. 1 . The modern two-step reaction $\left(n_{t h}, 2 \gamma\right)$ experiment revealed the existence of such a kind of dependence [16].

It was observed the smooth form of the function describing the energy spectra of the evaporated nucleons for the composite ${ }^{181} \mathrm{~W}$ nucleus for several different initial excitation energies [17]. But, analysis [18] indicates that in the excitation energy near the threshold of the second gap of the Cooper pair of nucleons, the partial widths of the nucleon emission increase many times, compared with the partial widths in neighboring excitation energies of the residual nucleus. This tendency does not change (or shows just a moderate variation) when the energy of the incident protons in the $(p, n)$ reaction is changed [18]. This can be explained only if the partial width of a nucleon emission is strongly dependent on the excitation energy of the residual nucleus and if the Strutinsky model for the level density [19] is used for the reproduction of the evaporate spectra. The set of parameters in the Strutinsky model approximation for the masses $40 \leq A \leq 200$ is derived from the level density obtained by $\left(n_{t h}, 2 \gamma\right)$ reaction. This means that, even for the different excitation energies of the produced nucleus ${ }^{182} \mathrm{~W}$, the product $\Gamma \cdot \rho$ preserves it's form. Moreover, in the case of $(p, n)$ reactions the wave function of the excited levels of the target nucleus (neutron resonance) changes significantly through the decay when evaporated neutron appears.

Therefore, it is possible to obtain the correct form of the energy dependence of the $\Gamma \cdot \rho$ product and, accordingly, the cross-section, for fixed nucleus excitation energy and for different energies of charged particles beam, even if the calculated values of $\Gamma$ and $\rho$ 
are not correct.

\subsection{Principles of the proposed modified model of radiation strength functions}

In the previous section we mentioned the effect on dependence of the partial width for a nucleon emission on the excitation energy of the residual nucleus. This effect opens a possibility for a modification of the standard form of the relation between the radiative strength function and the excited level density. The modified expression Eq. 1 describing the radiation strength of the gamma transitions between an arbitrary compound state $\lambda$ and any low-lying level $i$, can be written in the following form [20]:

$$
k_{\text {modif }}=k_{\text {sandard }} / D_{i}=\left(\Gamma_{\lambda i} /\left(E_{\gamma}^{3} A^{3 / 2} D_{\lambda}\right)\right) / D_{i}
$$

This modification takes into account the fact that the radiative strength function is dependent on the average spacing $D_{i}$ between the low-lying levels $i$.

In practice, in order to maintain continuity with Eq. 1, the following modification is suitable:

$$
k_{\text {modif }}=k_{\text {sandard }} \frac{D_{\text {asim }}}{D_{i}}=\frac{\Gamma_{\lambda i}}{E_{\gamma}^{3} A^{3 / 2} D_{\lambda}} \frac{D_{\text {asim }}}{D_{i}}
$$

In Eq. $3 D_{\text {asim }}$ is the asymptotic spacing between the levels of a heated nucleus treated as a pure fermion system (defined, for example, by non-interacting Fermi gas model) and $D_{i}$ is the maximum possible expected space between the intermediate levels for a given excitation energy. The specific value $D_{i}$ is the outcome of the coexistence and the interaction of the quasiparticle and the phonon types of excitations in the nucleus. Considering that the degree of fragmentation of some nuclear states is minimal at it's initial energy and grows up with energy increase [21], it can be expected that $D_{\text {asim }} \leq D_{i}$ and $k_{\text {modif }} \geq k_{\text {standard }}$ for the highest number of gamma transitions.

\section{Measurement of the two-step gamma cascade intensity of ${ }^{28} \mathrm{Al}$}

The ${ }^{28} \mathrm{Al}$ compound state gamma decay was measured in order to test the new modified model describing the dependence of the radiative strength function on the level density. The two-step gamma cascade method was used for this purpose.

It is commonly believed that the mechanism of neutron capture in light nuclei, for example, is significantly more dependent on the structure of the wave function of the excited level than in the neutron capture reaction in medium and heavy mass nuclei. Therefore, there is a modest practical interest for the average parameters of cascade gamma decay of neutron resonance in light nuclei. However, it can be important for the estimation of the reliability of the radiation strength functions for the gamma transitions between the levels of the heated nucleus obtained by the use of modified model Eqs. 2 and 3 .

The spectroscopic analysis data were already published and all details can be found in reference [22]. In this paper we present just a short description of the measurement procedure and the analysis of the spectroscopic information. 


\subsection{Experimental set-up}

The two-step cascades emitted after thermal neutrons capture in the ${ }^{27} \mathrm{Al}$ target were measured at the LWN-15 reactor in Rež, Czech Republic. Gamma-gamma coincidences were registered by two HPGe detectors with $28 \%$ and $25 \%$ relative efficiency, respectively, which have the standard energy resolution for this type of detectors. The time resolution was better than $10 \mathrm{~ns}$. The optimal count rate for the gamma-gamma coincidence detection of the ordinary fast-slow coincidences scheme was $100-200 \mathrm{~s}^{-1}$. This count rate is a trade-off between two demands: to get the maximal possible values of the photopeak underlying background intensity ratios and to collect at least several tens of thousand events for the most intensive peaks. The required result can be achieved if the duration of the experiment is about several days at least and if the mass of the target ranges from hundreds of milligrams to several grams.

\subsection{Spectroscopic information}

The coincidence events were analyzed with the standard method [1,2] based on the sum coincidence principle. Fig. 2 presents the most informative part of the measured spectrum.

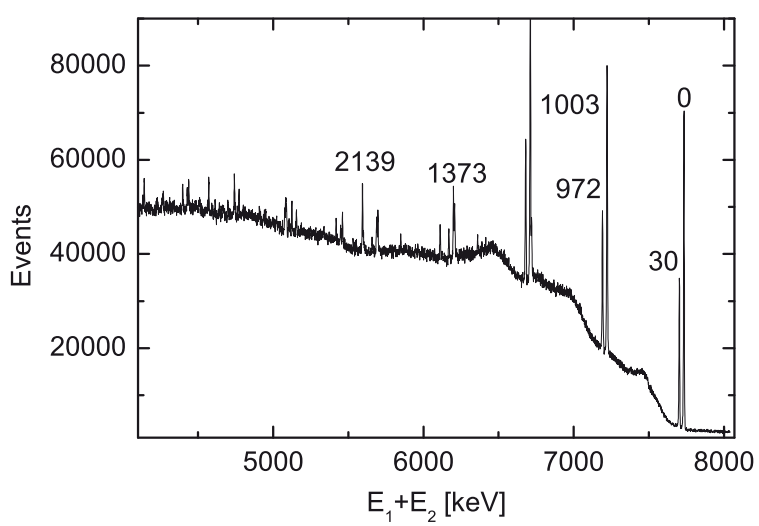

Figure 2: The main part of the sum coincidence spectrum of ${ }^{28} \mathrm{Al}$. Full-energy peaks are labeled with the energy (in $\mathrm{keV}$ ) of the final cascades levels.

Using the procedure described in [23], the intensity distributions of the cascades were obtained for some selected primary transition energy. For example, Fig. 3 shows the half of the measured intensity spectra of the cascades with energy of $7.725 \mathrm{MeV}$ populating the ground state of the ${ }^{28} \mathrm{Al}$ nucleus (for $E_{\gamma}<0.5 B_{n}$ ). The other half is mirror symmetric [24]. The transition energy and the intesity of about 250 cascades were obtained using 13 collected spectra, similar to the one presented in Fig. 2. The positions and the area of the peaks in the spectrum are uniquely identified by the cascade parameters. 


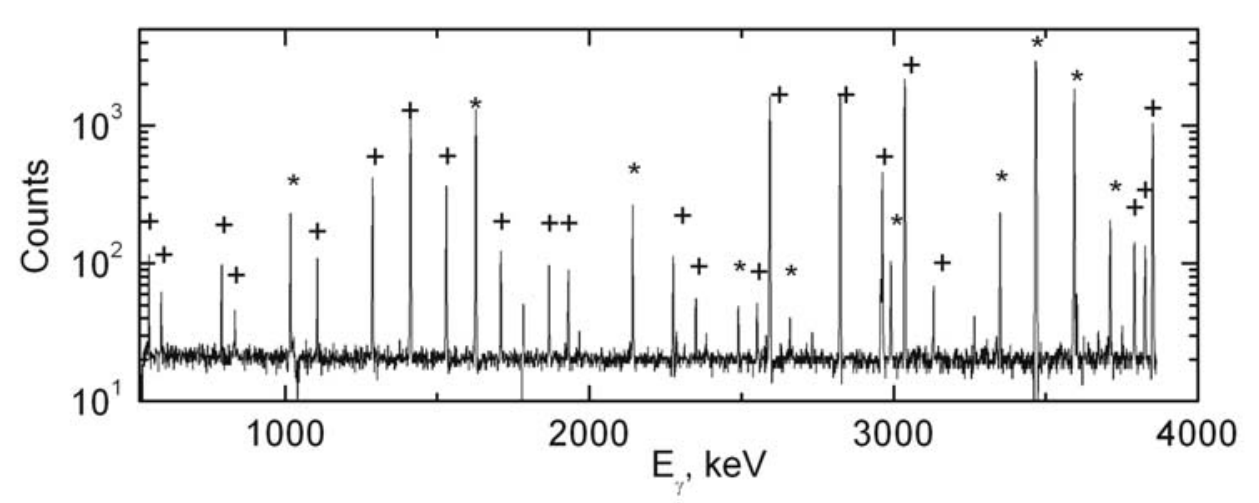

Figure 3: Half of the intensity distribution of the two-step cascades with energy 7.725 MeV populating the ground state. The primary transition of the ${ }^{28} \mathrm{Al}$ cascades are marked by crosses, whereas asterisks are the secondary one's. (The spectrum is shifted up to 20 counts due to the logarithmic scale.)

The order of the quanta in the cascades was determined by an algorithm [25]. This algorithm is based on a fact: the primary gamma transition has some energy in different two-step cascades and the second cascade photon energies have shifted on a difference for the final cascade level energies.

After the quanta sequence and the intermediate level energy were determined, almost all detected cascades were exactly placed into the decay scheme up to the energy of $\approx$ $B_{n}-520 \mathrm{keV}$. This method of two-step gamma cascades gives the posibility to provide the most complete level scheme for an investigated nucleus. The total number of levels observed with this method in ${ }^{28} \mathrm{Al}$ is close to hundred; file of evaluated data [26] contains about 45 levels identified up to now. It should be emphasized that in the two-step cascade spectrum registered after the thermal neutron capture, the number of observed excited levels is always noticeably higher than the number of levels found in any other nuclear spectroscopy method.

In order to extract the values of the level density and of the radiative strength function by the analysis of the experimental data, it is required to transform the relative intensities of the resolved peaks into absolute values (in \% per decay). This was done by normalization to the absolute intensities $i_{1}$ [27] of some primary transitions, multiplied by the branching ratios $B_{r}$ of the corresponding secondary transitions. $B_{r}$ was determined from the standard data treatment of the same measurement set of $\gamma-\gamma$ coincidence.

The dependence of the absolute intesity of the two-step gamma cascades on the energy of the primary gamma transition, was obtained as final spectroscopic result. This result is crucial for the determination of the level density and of the radiative strength function. Fig. 4 presents the absolute intensity of the two-step gamma cascades on the two first excited levels and the ground level [22]. The values of the level density and of the radiative strength function for ${ }^{28} \mathrm{Al}$ were obtained by the fitting procedure described in references $[2,20]$ and the spectroscopic results are presented on Fig. 4. 


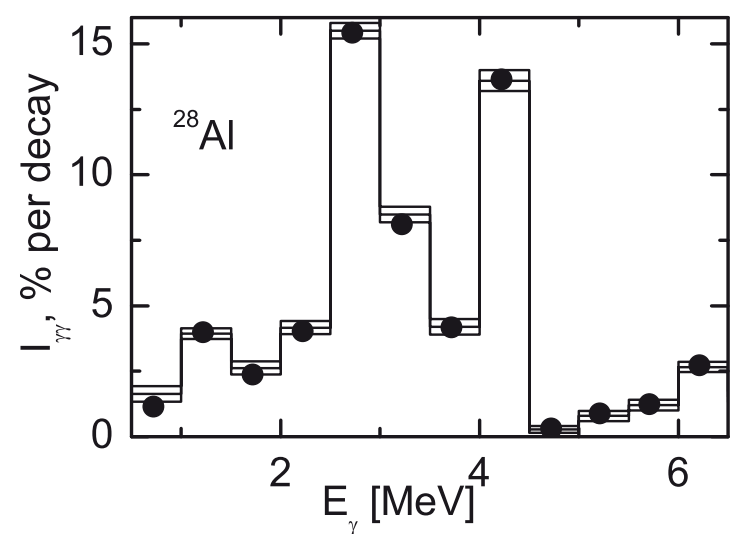

Figure 4: Histogram - distribution of the intensity of the two - step gamma cascades of the ${ }^{28} \mathrm{Al}$ to the ground, the first and the second excited level as functions of their primary transition energy. The approximated values $I_{\gamma \gamma}$ obtained by one of the variants defining the random radiative strength and the level density functions are noted by dots.

\subsection{The levels density and the radiation strength functions of the ${ }^{28} \mathrm{Al}$ nucleus}

The unambiguous determination of $\rho$ and $\Gamma$ from the measured spectra is impossible, even in principle, because the functional dependence of the two-step gamma cascade intensity on the level density and on the radiative strength function is nonlinear and degenerated:

$$
I_{\gamma \gamma}\left(E_{1}\right)=\sum_{\lambda, f} \sum_{i} \frac{\Gamma_{\lambda i}}{\Gamma_{\lambda}} \frac{\Gamma_{i f}}{\Gamma_{i}}=\sum_{\lambda, f} \frac{\Gamma_{\lambda i}}{\left\langle\Gamma_{\lambda i}\right\rangle m_{\lambda i}} n_{\lambda i} \frac{\Gamma_{i f}}{\left\langle\Gamma_{i f}\right\rangle m_{\mathrm{i} f}}
$$

where $\Gamma_{\lambda i}$ and $\Gamma_{i f}$ are the partial radiative widths corresponding to the primary and to the secondary transition; $n_{\lambda i}=\rho_{\lambda} \Delta E_{i}$ is the number of the excited intermediate levels in a certain interval of the excitation energy $\Delta E_{i} ;\left\langle\Gamma_{\lambda i}\right\rangle$ and $\left\langle\Gamma_{i f}\right\rangle$ are the average values of the corresponding intervals of the nucleus excitation energy widths; $m_{\lambda i}$ and $m_{i f}$ are the number of levels in the same intervals.

However, the form of the functional relation for the parameters appearing in Eq. 4 limits the region of their possible parameter values. For this reason $N$ values of the experimental cascade intensities always can be converted in $\sim 2 N$ values of $\rho$ and $\Gamma$, satisfying the conditions:

$$
\begin{gathered}
\rho_{1} \leq \rho \leq \rho_{2} \\
\Gamma_{1} \leq \Gamma \leq \Gamma_{2} .
\end{gathered}
$$

With the use of an iterative technique it is possible to obtain a random function of the level density and of the radivative strength function which can reproduce, with high precision, the experimental values of $I_{\gamma \gamma}$. Hence the measurement of the two-step gamma cascades provides a good possibility to determine the most probable values for these parameters. 
The approximated values of the measurement $I_{\gamma \gamma}$ (obtained by the iterative method based on Eq. 4) are presented by the dots in Fig. 4. These values correspond to any of the single random functions of the level density and of the radiative strength function. In Fig. 5 the thin lines represent the obtained random functions for the level density and by the dots are shown the average value of these random functions. The most probable region of the level density is presented on Fig. 6.

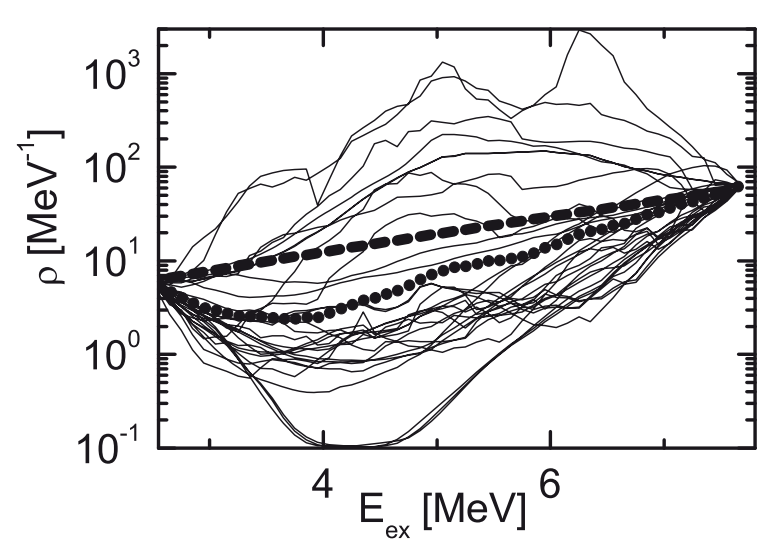

Figure 5: A set of random functions describing level densities able to reproduce data presented on Fig. 4 with a very close and small $\chi^{2}$ values (thin lines). The average value of the entire set of random functions is presented by dots. Dashed line - model value for $\rho$ [29].

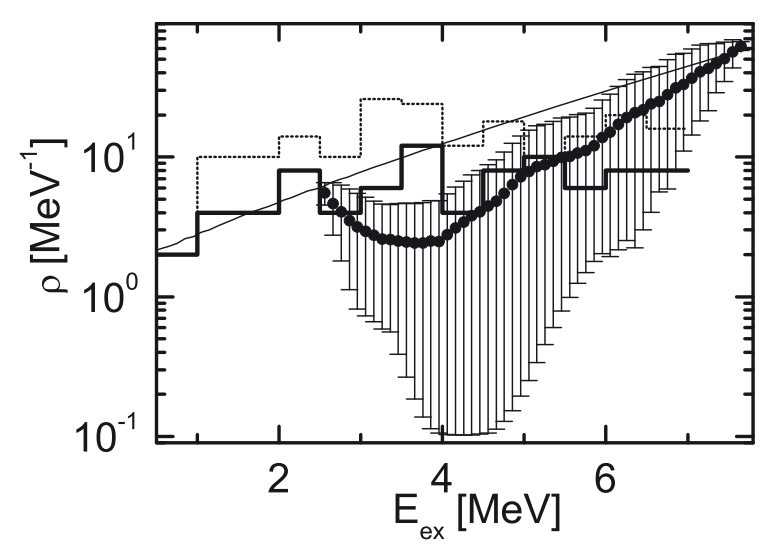

Figure 6: Thick line - model value for $\rho$ [29]. Solid histogram presents density of the ${ }^{28} \mathrm{Al}$ levels from [26], dotted histogram - from processing spectra [22] similar to those shown in Fig. 1. Dots with error bars are the results of this study (same as in Fig. 5).

Two different forms of the radiative strength function dependence on the level density (Eq. 1 and 3) were used in the fitting procedure. In this way, the modified dependence in 
Eq. 3 is tested in comparison with the standard one in Eq. 1 on the set of experimental data presented in Fig. 4. In Figs. 7 and 8 are presented the obtained random functions and the most probable region of the radiation strenght function when using Eq. 1. The same results are shown in Figs. 9 and 10, but here Eq. 3 was used. The difference between the obtained values of the radiative strength function, as presented in Figs. 8 and 10, provides the information about the influence of the collective enhancement for the level density on the radiative strength function.

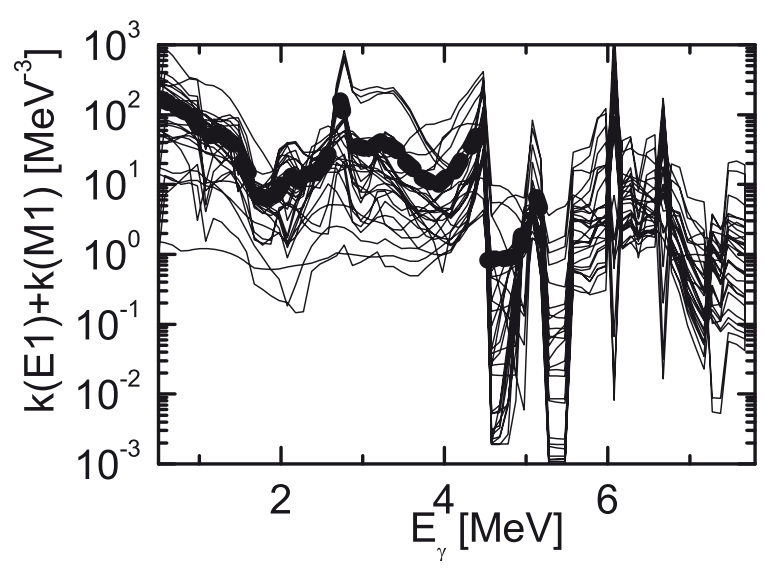

Figure 7: Thin lines - set of random functions (obtained by interative process)describing the radiation strength function in standard definition Eq. 1 able to reproduced data presented on Fig. 4. Dotted line - average for sum of strength function E1- and M1- transition.

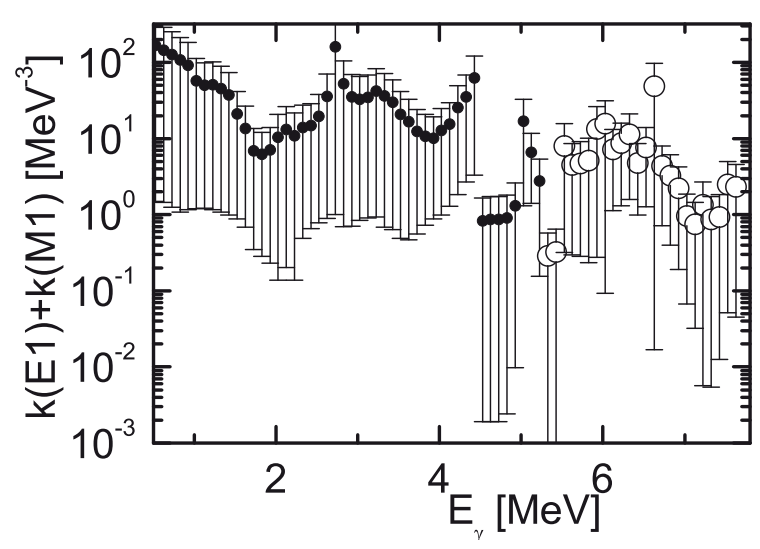

Figure 8: Values for the radiation strenght function in standard definition Eq. 1. Dark dots - sum of M1- and E1-transition. Open dots - data only for M1-transition.

The values of the radiative strength functions for the $E 1+M 1$ transition (dotted line) and the values for only the $M 1$-transition are presented in Figs. 7 and 8 . In the energy region above $3.465 \mathrm{MeV}$ are present only M1- transitions. The positive parity 
of the ${ }^{28} \mathrm{Al}$ neutron resonances and of all the known low-lying levels define practically only $M 1$ multipolarities of the primary transitions. Hence, the information on the $E 1$ radiative strength functions for $E_{\gamma}>4260 \mathrm{keV}$ cannot be obtained from the two-step cascade intensity.

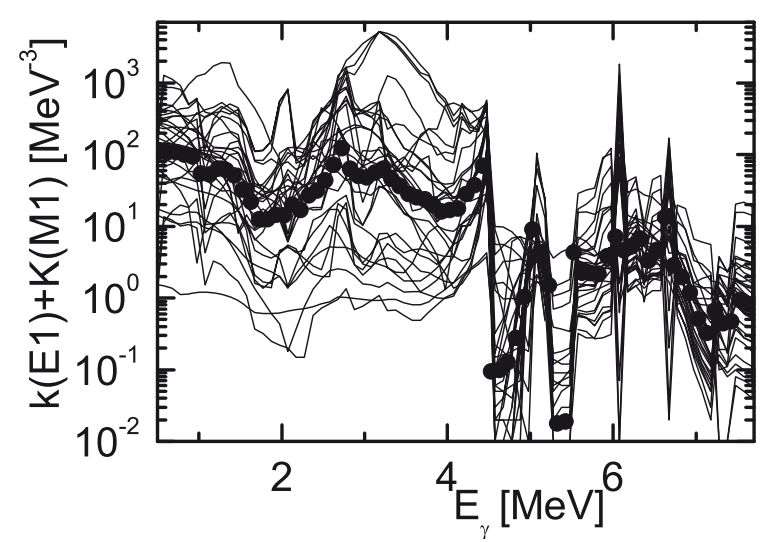

Figure 9: The same as in Fig. 7 for the modified model of the radiation strength function Eq. 3. Dotted line is average values.

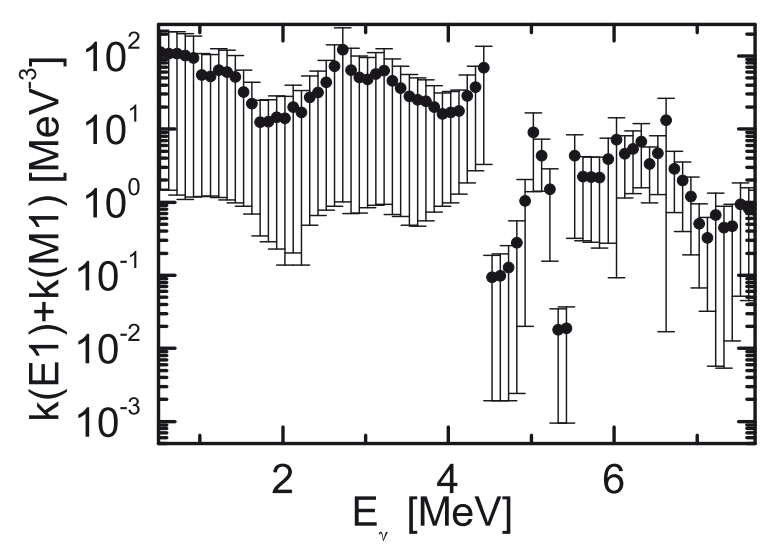

Figure 10: Average values of radation strenght function with error bars in case of modified model Eq. 3.

The thermal neutrons capture cross sections for this nucleus is determinated by the resonances with spins 2 and 3 . The dominant dipole type of the primary gamma-transitions limits the spin window for the intermediate levels to the interval $1 \leq J \leq 4$. The level density in this spin interval, calculated from the schemes evaluated from file $[26,27]$ in Fig. 5 is compared with similar data obtained from the two-step cascades. The corresponding data, together with the density of the neutron resonances are always used for the normalization of the relative level density in both the one- and the two-step reactions. Unfortunately, 
the independent analysis [18] of the accuracy for the values $D_{\lambda}$ showed that the possible systematical error may underestimate the density of the neutron resonances, even by one order of magnitude.

The influence of the mentioned error of the level density in all excitations can be accounted by a simple change of the normalization parameters for the set of random chosen functions describing the level density and the radiative strength. However, no data related to the real errors on the neutron resonances densities, identified in [28], are available in the literature until now.

\section{Discussion}

The unknown values of $\Gamma$ and $\rho$ correspond to each interval of the excitation energy for the intermediate levels (two-step cascades, in particular). In the general case, the inequality of the radiative strength functions for the primary and the secondary gamma transitions should be taken into account, even if they have the same energy and multipolarity. In principle, these circumstances make degenerated any system of nonlinear equations which connect the spectra intensity and unknown gamma-decay parameters. However, even in such case it can be defined a region Eq. 5 of the possible values for the level density and for the radiation strength functions. Authors of [2] showed that this can be done with acceptable precision if a sufficiently large set of values of the random functional dependencies for $\rho$ and $\Gamma$ on the energy gamma-transition are provided for excitation of nuclei. This is true in the frame of the postulate that the difference between the average of the random values and true unknown real value always tends always to minimum. Therefore, the value obtained in the two-step gamma cascads experiment [2] can be considered as a valid results, with the corresponding uncertainty of the level density and the radiation strength functions values.

The very significant difference of the ${ }^{28} \mathrm{Al}$ low-lying levels density in the region from $\rho \approx 4 \mathrm{MeV}^{-1}$ up to $\rho \approx 20 \mathrm{MeV}^{-1}$ (Fig. 5, solid and dotted histogram), can explain the skepticsm related to the contemporary experiment. This includes as well the occurrence of the possible methodical errors in the determination of the values of $\Gamma$ and $\rho$. However, the hypothesis in Eq. 3 can be taken as a first approximation of the model description for the radiative strength functions in any heated nucleus. As a special case, this hypothesis includes the existing assumption that the radiative strength functions and the level density for any nucleus are independent from the structure of the excited levels. This possibility should be checked on a large set of experimental data.

The practical absence of the negative-parity levels below $3.3 \mathrm{MeV}$ does not allow to obtain the strength function of the $E 1$-transition in this interval for ${ }^{28} \mathrm{Al}$ excitation energies. It is also not possible to obtain a satisfactory approximation for the intensity distribution in the range of the primary cascade energies from $E_{1} \approx 0.5 \mathrm{MeV}$ to $E_{1} \approx 1 \mathrm{MeV}$. The increase of the strength functions in this interval, which can be observed in Fig. 8 and 10, can be explained as a consequence of the absence of secondary cascade transitions enhancement in this interval of energies. The corresponding increase in the strength functions can be qualitatively explained only by the presence of the collective type of primary transitions in the region of $B_{n}$ and the corresponding vibration enhancement of the level density. This might be possible if the breaking threshold of the next Cooper pair falls randomly in the region of nuclear excitation near $B_{n}$. 
In Fig. 11, we show the potential effectiveness of the technique presented in this work. In Fig. 11a, the level density was determined by model [29], whereas in Fig. 11b the level density was obtained with the approximation similar to the one presented on Fig. 5 but assuming equality of $\rho$ for the levels of positive and negative parity. The main result of this exercise is to show that an independent experiment, able to determine the level density, will make possible to obtain a precise information on the radiation strength function. In the same way an experiment designed to determine the radiative strength function can provide the values of the level density.
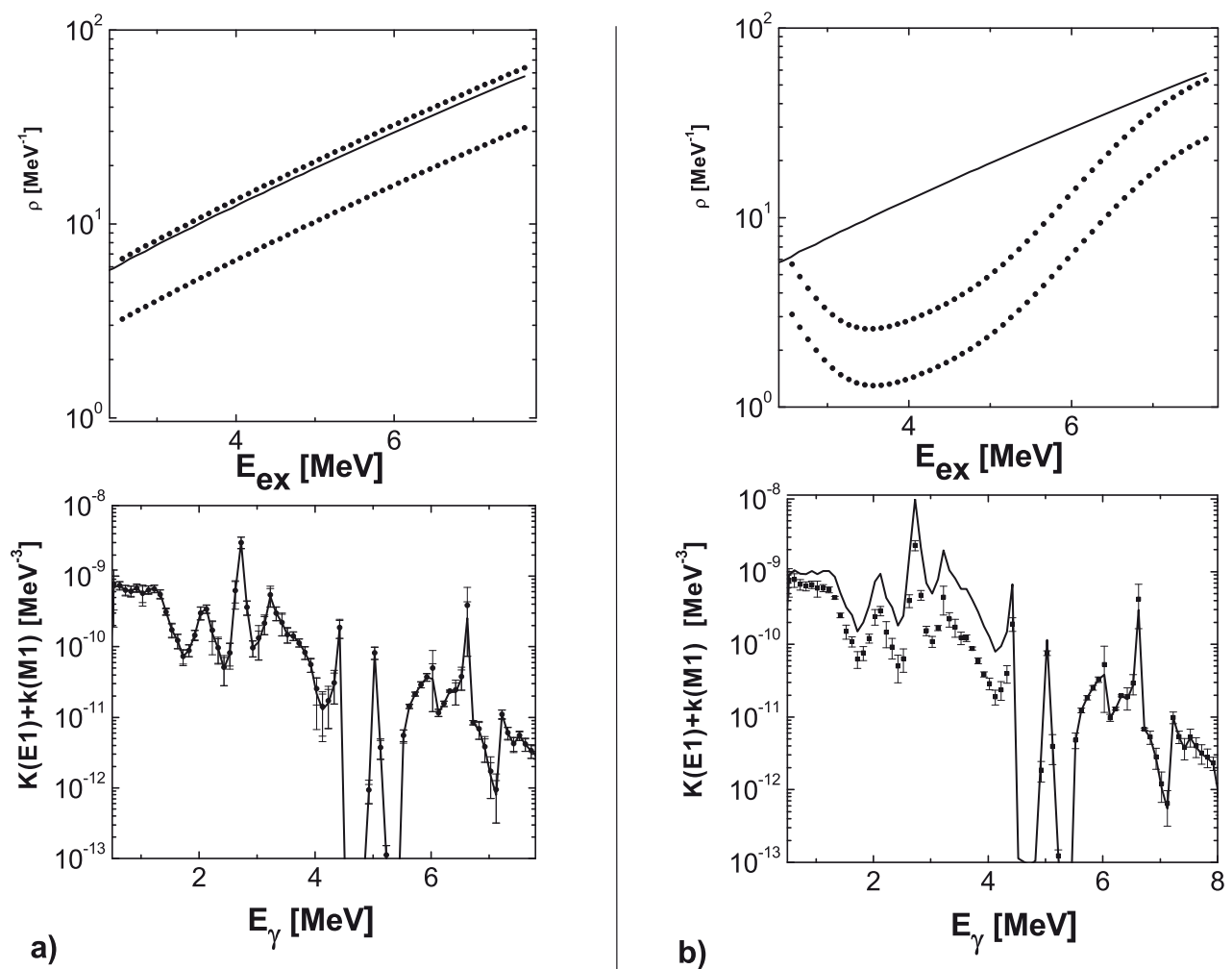

Figure 11: a) Top row: fixed to the level density of ${ }^{28} \mathrm{Al}$ (dots and line) from model [29]. Bottom row: the best approximation of the radiative strength functions for fixed level density. Points with error bars: standard presentation of the radiation strength function; line: strength function in the Eq. 3. b) Top row: level density from model [29] (line) and approximated level density (dots). Bottom row: points with error bars: standard presentation of the radiation strength function; line: strength function in the Eq. 3.

Searching the solution of a degenerate nonlinear systems of equations can always give as a result both the real function and a local maximum likelihood function. Methods good enough to identify and solve such systems have already been developed. The data in Fig. 4, presenting the worst version for the fitting process of the experimental data at $I_{\gamma \gamma}$, has been used to determine $\Gamma$ and $\rho$. Hence, the number of iterations required to achieve a minimum disagreement between the experimental and the approximation values for the cascade gamma decay of the neutron resonances on ${ }^{27} \mathrm{Al}$ nucleus usually exceeds $10^{5}$ for 
each variant of the calculation. Here, as in many other cases, it is convenient that the first iteration includes in source data specific values of the strength functions for the most intensive gamma-transition "spread" under appropriate intervals of the primary gammatransition energy.

\section{Conclusion}

In this work we proposed Eqs. 2 and 3 as new hypothesis for the energy dependence form of the radiative strength function for the excited level density of the residual nuclei. This hypothesis was experimentally tested on the light nuclei ${ }^{28} \mathrm{Al}$. The spectrum of the random functions for the level density as well as the radiative strength functions of the dipole E1- and $M 1$-transitions were determined for ${ }^{27} \mathrm{Al}$. The obtained functions can reproduce very precisely the intensity of the two-step cascade following the radiative capture of thermal neutrons for a given energy of primary transitions. The density of the intermediate levels corresponding to all the energetically resolved cascades observed (including those firstly established in reaction $\left(n_{t h}, 2 \gamma\right)$ ) can be reproduced correctly using the mean value of the obtained functions for the level density. In this work, for the first time, we obtained the information about the influence of the collective enhancement of the level density on the radiative strength function.

The results of the ${ }^{28} \mathrm{Al}$ two-step cascades experiment show that the hypothesis given by Eq. 2 can ensure the maximum precision for the description of the spectra and of the cross sections in a broad region of nuclear masses [20] (including light nuclei). Unfortunately, the determination of the density for the low-lying levels and the neutron resonances in a custom nucleus, with acceptable precision, still remains an unsolved problem. For this reason the dynamic model of the interaction of superfluid and normal states of nuclei, in the transition region from levels with a simple wave function to extremely complex compound-states, can not be identified and described correctly.

Further experiments are indispensable to accomplish this task. This can be done through the implementation of multi-step reactions. The most promising technique is the measurement of the intensity for a sequence of three or more cascade photons [30] in the radiation capture of nucleons and light nuclei reactions, as well as measuring the intensities of the cascades, containing nucleon products of nuclear reactions [9], in coincidences with photons.

The improvement of the process for the determination of the radiative strength function and the level density can be achieved also by the development of new models. These models should introduce the dependence of both the radiative strength function and the level density on the same fitting parameters. The first of all these parameters should be the threshold gap for the Couper pair and the mutually connected coefficients for vibration and collective enhancement of the levels density. This should be done for the radiative strength functions at decay of the levels with large enough components of the phonon type in the structure of their wave functions. For example, it can be expected that the models of this type will be able to easily reproduce the intensity of the cascades primary transition in the $\approx 0.5-1 \mathrm{MeV}$ energy range (Fig. 2 ) for ${ }^{28} \mathrm{Al}$, as well as, for large number of other nuclei. Under favorable conditions (a small number of parameters), it can be expected a rather uniquely determination of the radiative strength functions and of the level density 
even without fixing one of those parameters.

\section{References}

[1] S. T. Boneva, E. V. Vasileva, Yu. P. Popov, A. M. Sukhovoj and A. Khitrov, Sov. J. Part. Nucl. 22, 232 (1991).

[2] S. T. Boneva, E. V. Vasileva, Yu. P. Popov, A. M. Sukhovoj and V.A. Khitrov, Sov. J. Part. Nucl. 22, 698 (1991).

[3] H. Vonach, Phys. Rev. C 38, 2052-2062 (1988).

[4] E. V. Vasilieva, A. M. Sukhovoj and V. A. Khitrov, Phys. At. Nucl. 64, 153 (2001).

[5] A. M. Sukhovoj and V. A. Khitrov, Phys. Particl. and Nuclei 36, 359 (2005).

[6] B. V. Zhuravlev, Bull. Rus. Acad. Sci. Phys. 63, 123 (1999).

[7] G. A. Bartholomew, E. D. Earle, A. J. Ferguson, J. W. Knowles and M. A. Lone, Advances in nuclear physics 7, 229 (1973).

[8] A. Schiller, L. Bergholt, M. Guttormsen, E. Melby, J. Rekstad and S. Siem, Nucl. Instrum. And Methods A 447, 498 (2000).

[9] A.M. Sukhovoj and V. A. Khitrov, Nucl. Instrum. Phys. At. Nucl. 73, 1635 (2010).

[10] A. M. Sukhovoj and V. A. Khitrov, In Proc. of the XVII International Seminar on Interaction of Neutrons with Nuclei, Dubna (2009).

[11] A. C. Larsen, M. Guttormsen, R. Chankova, F. Ingebretsen, T.Lonnroth, S. Messelt, J. Rekstad, A. Shiller, S. Siem, N. U .H. Syed and A. Voinov, Phys. Rev. C 76, 044303 (2007).

[12] A. C. Larsen, M. Guttormsen, M. Krtcka, A. Betak, A. Burger, A. Gorgen, H. T. Nyhus, J. Rekstad, A. Schiller, S. Siem, H. K. Toft, G. M. Tveten, A. V. Voinov and K.Wikan, Phys. Rev. C 83, 034315 (2011).

[13] A. M. Sukhovoj, V. A. Khitrov and W. I. Furman, Phys. At. Nucl. 72, 1759 (2009).

[14] V. A. Khitro,v and Li Chol and A. M. Sukhovoj, Proceedings of the XII International Seminar on Interactions of Neutrons with Nuclei, Dubna, E3-2004-9, (2004).

[15] V. G. Soloviev, Sov. Phys. Part. Nuc. 3, 390 (1972).

[16] A. M. Sukhovoj and V. A. Khitrov, Phys. Particl. and Nuclei 37, 899 (2006).

[17] V. G. Pronyaev et al., Sov. J. Nuc. Phys. 30, 310 (1979).

[18] A. M. Sukhovoj and V.A. Khitrov, Phys. At. Nucl. 73, 1507 (2010). 
[19] V. M. Strutinsky, Proceedings of the International Congress on Nuclear Physics, Paris, 617 (1958).

[20] N. Jovancevic, A. M. Sukhovoj, W. I. Furman and V. A. Khitrov, Proc. XX International Seminar on Interaction of Neutrons with Nuclei, Alushta (2012).

[21] L. A. Malov and V. G. Solovev, Yad. Phys. 26, 729 (1977).

[22] J. Honzatko, V.A. Khitrov, A.M. Sukhovoj and I. Tomandl, Fizika B 12, 299 (2003).

[23] S. T. Boneva, V. A. Khitrov and A. M. Sukhovoj, Nucl. Phys. A 12, 199 (1995).

[24] A. M. Sukhovoj and V. A. Khitrov, Instrum. Exp. Tech. 27, 1071 (1984).

[25] Yu. P. Popov et al., Bull. Acad. Sci. USSR Phys. Ser. 48, 53 (1984).

[26] NNDC, Evalueted Nuclear Structure Data File (2013), URL http://www.nndc.bnl.gov/nndc/ensdf

[27] IAEA, Evalueted Gamma-ray Activation File (2013), URL http://wwwnds.iaea.org/pgaa/egaf.html

[28] A. M. Sukhovoj and V.A. Khitrov, XVIII International Seminar on Interaction of Neutrons with Nuclei, Dubna (2011).

[29] W. Dilg, W. Schantl, H. Vonach and M. Uhl, Nucl. Phys. A 217, 269 (1973).

[30] K. Furutaka, M. Oshima, A. Kimura, Y. Toh, M.Koizumi, T. Kin and J.Goto,In Proceedings of the International Conference on nuclear data for science and technology 2007, Nice, 517 (2007). 